Jaap van Milgen ${ }^{1,2}$, Mathieu Gloaguen ${ }^{1,2}$, Nathalie Le Floc ${ }^{1} h^{1,2}$, Ludovic Brossard ${ }^{1,2}$, Yvan Primot ${ }^{3}$, Etienne Corrent ${ }^{3}$

'INRA, UMR1348 PEGASE, Saint-Gilles, France, ${ }^{2}$ Agrocampus Ouest, UMR1348 PEGASE, Rennes, France, ${ }^{3}$ Ajinomoto Eurolysine S.A.S., Paris, France

Introduction and objective

- Recent experimental evidence suggests that Val is the next-limiting amino acid for pigs in cereal-soybean meal based diets (after Lys, Met, Thr, and Trp, but before lle).

t-Val is now a

mulation of diets with a very

Detive: perform a meta-analysis of available data on the response of growing pigs to the Val supply.

\section{Data and statistical analysis}

28 dose-response studies with at least 4 levels of Val were obtained from 20 publications (9 of which were peer-reviewed).

The composition of feed ingredients (Sauvant et al., 2004) was used to calculate the standardized ileal digestibility (SID) and nutrient composition.

- Data were standardized (Figures 1 and 2):

relative to the NRC (1998) Val requirement estimate (as a \% in the diet or relative to Lys, depending on the design of the study)

relative to the response at the highest level of Val supplementation

Figur 1. Meta-design of the study. The horizontal line indicates the duration of the study (body weight range) and the vertical line
the range in Val contents \% of the NRC (1998) value).
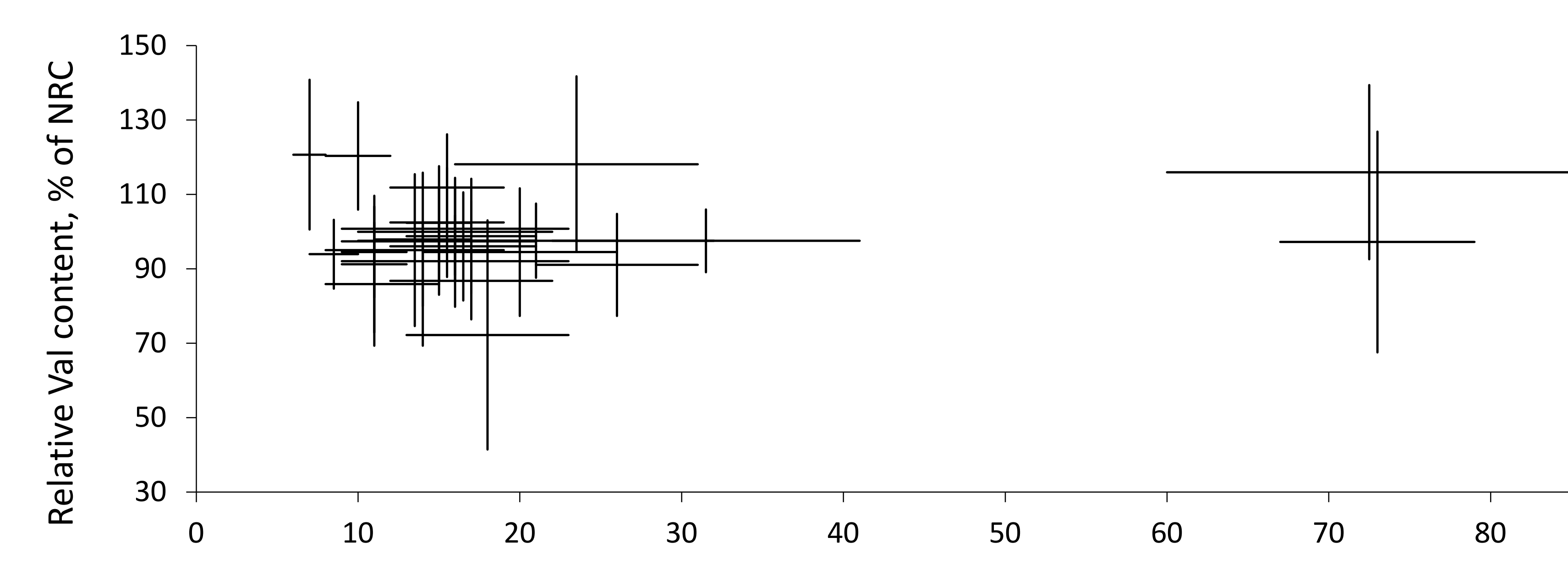

$\begin{array}{rl}40 & 50 \\ \text { Body weight, } \mathrm{kg}\end{array}$

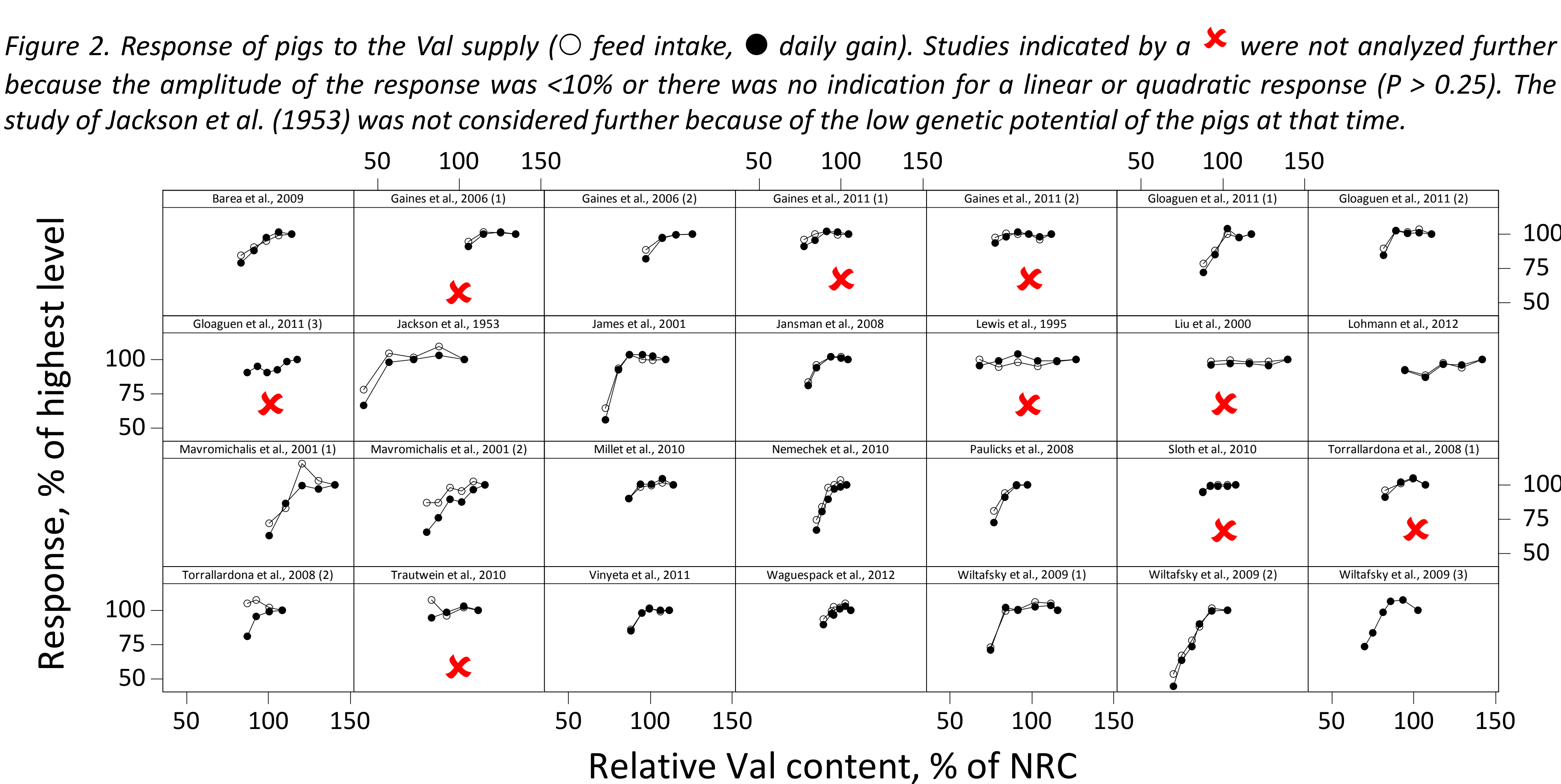

Figure 3 . A bent-stick model was used to account for the decline in Val requirement during the experiment. The green area (left
panel) Illustratest that, in a dose-response experiment, a partial response will be observed for some of the treatments beccause the

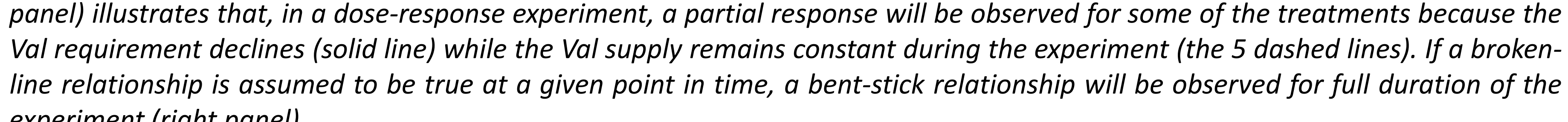
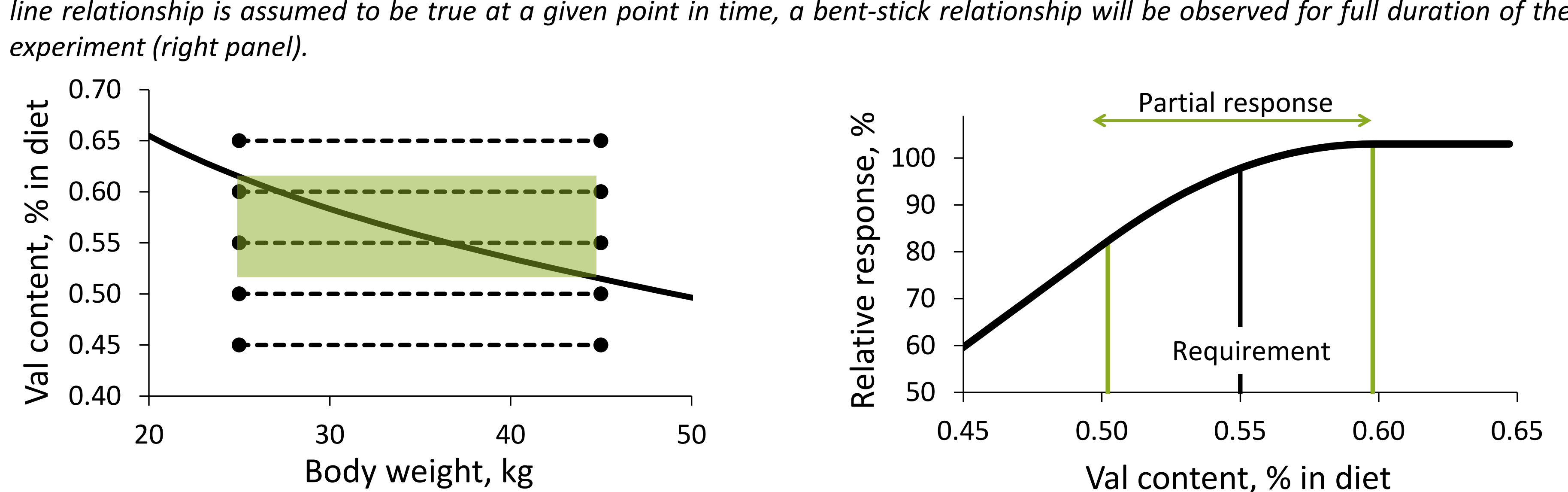

The bent-stick model was parameterized using feed intake and daily gain as response criteria with the following model parameters:

- a plateau value for each study

- a Val requirement for each study (i.e., the mid-point of the transition phase)

- a common slope across studies

The duration of the partial response was fixed for each study based on the anticipated decline in the Val requirement during the experiment.

\section{Results}

- The responses of feed intake and daily gain to the Val supply were very similar (Figure 2). Val requirement estimates ranged from $87-117 \%$ of the NRC (1998) value for feed intake, and from $84-114 \%$ for daily gain $(94 \%$ on average, which corresponds to $64 \%$ SID Val:Lys).

Increasing the SID Val:Lys supply from $64 \%$ to $69 \%$ resulted in a $5 \%$ performance

A $10 \%$ reduction in Val supply below the requirement reduced feed intake and growth by respectively $23 \%$ and $27 \%$

Hith increasing amino acid or protein level (Figure 4 and Table 1):

Figure 4. Relation between the Val requirement estimate
and the Phe content in the diet. Both values are expressed and the Phe content in the diet. Both values are expressed
as $a$ percentage of the NRC (1998) requirement

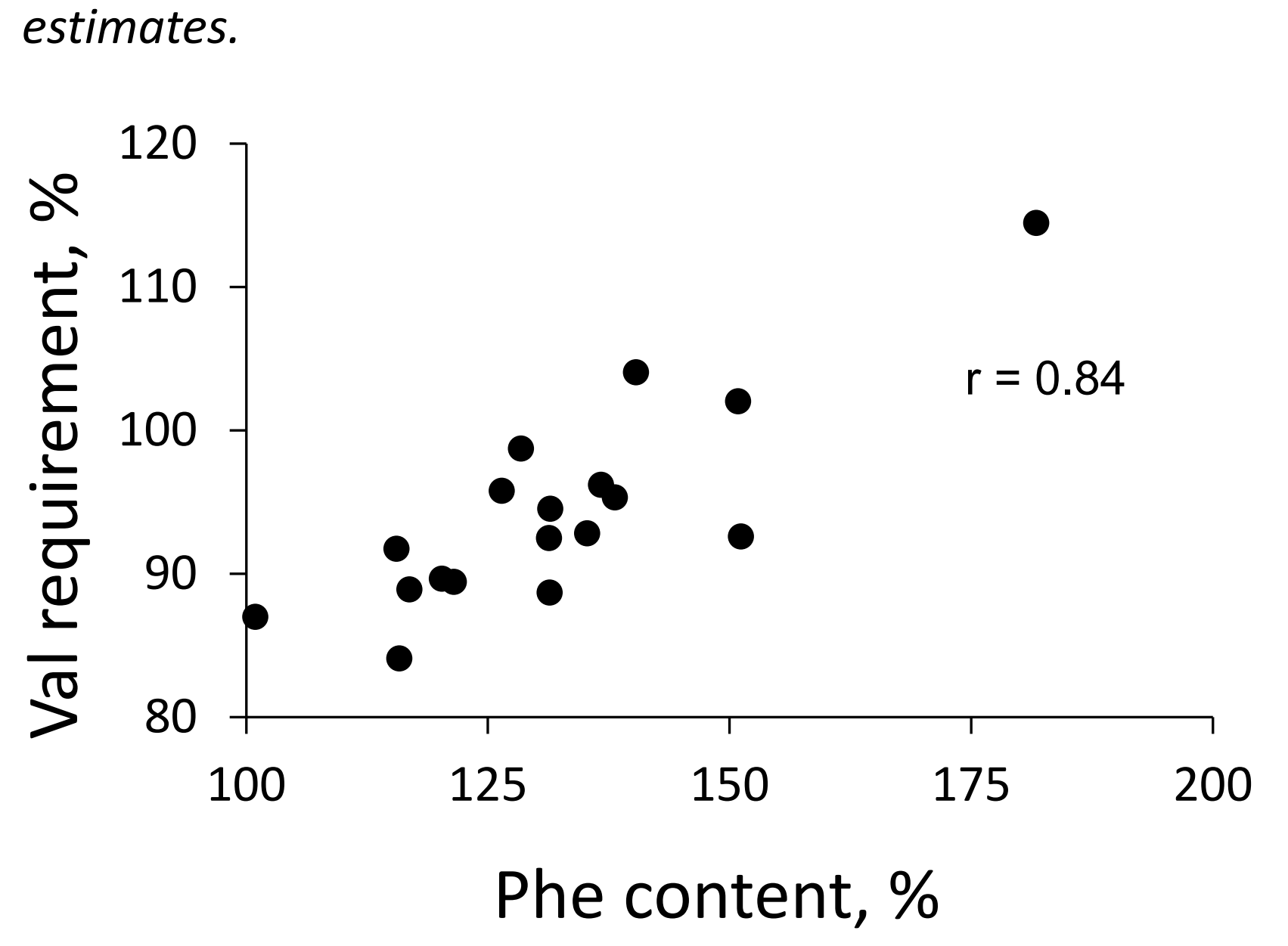

Table 1. Correlation coefficients between the val
requirement estimate and the amino acid content in the
diet. \begin{tabular}{l|l|l|l|l|l|l}
\hline Amino acid & $\mathrm{r}$ & Amino acid & $\mathrm{r}$ \\
\hline & 0.67 & Phetyr & 0.79
\end{tabular} \begin{tabular}{l|c|lc} 
Lys & 0.67 & Amino acid & $r$ \\
\hline Phe+Tyr & 0.79
\end{tabular} $\begin{array}{llll}\text { Met } & 0.43 & \text { Leu } & 0.47\end{array}$ $\begin{array}{llll}\text { Met+Cys } & 0.48 & \text { lle } & 0.17\end{array}$ $\begin{array}{llll}\text { Trp } & 0.51 & \text { His } & 0.75\end{array}$ $\begin{array}{llll}\text { Trp } & 0.51 & \text { His } & 0.75 \\ \text { Thr } & 0.68 & \text { Arg } & 0.06\end{array}$ $\begin{array}{ll}\text { Thr } & 0.68 \\ \text { Phe } & 0.84\end{array}$ Phe content, $\%$

\section{Conclusions}

Most of the studies on Val requirements have been carried out in pigs weighing $<35 \mathrm{~kg}$ Most of the response to a Val deficiency occurs through a reduction in feed intake. .

- The denciency

have an impact of the Val requirement. 